\title{
DOMAIN SIZE DISTRIBUTION OF Y-TZP NANO-PARTICLES USING XRD AND HRTEM
}

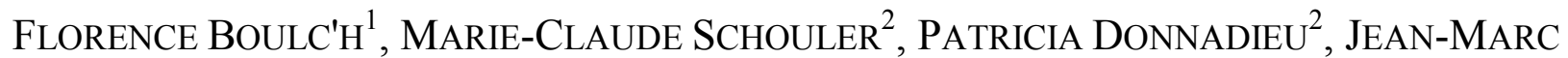 \\ CHAIX $^{2}$ AND ELISABETH DJURADO ${ }^{1}$
}

${ }^{1}$ Laboratoire d'Electrochimie et de Physico-Chimie des Matériaux et des Interfaces (LEPMI), ${ }^{2}$ Laboratoire de Thermodynamique et Physico-Chimie Métallurgiques (LTPCM), BP 75- 38402 Saint Martin d'Hères, France e-mail: Florence.Boulch@lepmi.inpg.fr

(Accepted August 27, 2001)

\begin{abstract}
Yttria doped nanocrystalline zirconia powder was prepared by spray-pyrolysis technique. Powder crystallized into tetragonal form, as dense and compositionally homogeneous polycrystalline spheres. X-Ray diffraction (XRD) and high resolution transmission electron microscopy (HRTEM) have been used in order to characterize the mean size and the size distribution of crystalline domains. An average size of $6 \mathrm{~nm}$ was calculated by Scherrer formula from X-Ray diffraction pattern. The domain size, determined by analysis method developed by Hytch from HRTEM observations, ranges from 5 to $22 \mathrm{~nm}$ with a main population around the value $12 \mathrm{~nm}$. Limits and complementary nature of XRD and HRTEM methods are discussed.
\end{abstract}

Keywords: HRTEM, image analysis, phase image, tetragonal zirconia, XRD.

\section{INTRODUCTION}

Yttria doped nanocrystalline zirconia (Y-Tetragonal Zirconia Polycrystals - referred to as $\left.2.5 \mathrm{Y}_{2} \mathrm{O}_{3}-\mathrm{ZrO}_{2}\right)$ is considered a potential candidate to be used as electrolyte in solid oxide fuel cells, due to its remarkable mechanical and thermal properties, compared to the cubic form, used up to now. The study of Y-TZP electrical properties is in progress (Boulc'h et al., 2001.) in order to compare electrical performance of tetragonal and cubic forms.

The high temperature, tetragonal form zirconia $\left(\mathrm{ZrO}_{2}\right)$ can be stabilized at room temperature provided a nanometric crystalline domain size is maintained (Djurado et al., 2000) and/or different oxides such as rare-earth oxides are added (Tsukuma et al., 1984). Unfortunately, metastable tetragonal phase may transform in the monoclinic form $(t \rightarrow m)$. Now, monoclinic zirconia presents low ionic conductivity and can not be viewed as electrolyte for solid oxide fuel cells. Therefore, the parameters influencing the $t \rightarrow m$ transformation are to be studied. On one hand, in undoped nanocrystalline zirconia, the martensitic $\mathrm{t} \rightarrow \mathrm{m}$ transformation was observed up to a critical size, evaluated to $25 \mathrm{~nm}$ (Djurado et al., 2000). On the other hand, depending on the crystalline domain size distribution, the $\mathrm{t} \rightarrow \mathrm{m}$ transformation occurs abruptly or continuously. Therefore, the final aim of the study is to correlate yttria doped and undoped nanocrystalline zirconia behaviors towards the martensitic transformation, in terms of size and size distribution. In a first time, X-Ray diffraction (XRD) and high resolution transmission electron microscopy (HRTEM) methods were used in order to measure the crystalline domains size and size distribution of $2.5 \mathrm{Y}_{2} \mathrm{O}_{3}-\mathrm{ZrO}_{2}$ powder.

The point of the present work is to present both methods and take benefits of their complementary nature in order to characterize the $2.5 \mathrm{Y}_{2} \mathrm{O}_{3}-\mathrm{ZrO}_{2}$ powder.

\section{MATERIALS AND METHODS}

$\left(\mathrm{ZrO}_{2}\right)_{0.975}\left(\mathrm{Y}_{2} \mathrm{O}_{3}\right)_{0.025}$ - referred to hereafter as $2.5 \mathrm{Y}_{2} \mathrm{O}_{3}-\mathrm{ZrO}_{2}$ - powder was synthesized by the spray-pyrolysis technique using an ultrasonic atomizer (Djurado and Meunier, 1998). The precursor solution was made up of a stoichiometric mixture of zirconyl nitrate hydrate $\left(\mathrm{ZrO}\left(\mathrm{NO}_{3}\right)_{2}\right.$, Aldrich) and yttrium nitrate $\left(\mathrm{Y}\left(\mathrm{NO}_{3}\right)_{3}\right.$, Aldrich) in distilled water. The concentration was $2.5^{*} 10^{-2} \mathrm{~mol}$. $\mathrm{L}^{-1}$. The solution was atomized by a $1.7 \mathrm{MHz}$ high-frequency ultrasonic mist generator. The gaseous $\mathrm{N}_{2}+\mathrm{O}_{2}$ mixture carried the aerosol at $6 \mathrm{~L} \mathrm{~min}^{-1}$ through a tubular furnace (Carbolite TZF - $60 \mathrm{~mm}$ in diameter and $1090 \mathrm{~mm}$ in 
heated length) heated at $600^{\circ} \mathrm{C}$. One advantage of this one-step synthesis is to obtain nanocrystalline powder of zirconia at low temperature in which no amorphous phase was detected with either XRD or with Raman spectrometry. Microscopic scanning observations show smooth submicronic spherical particles more or less agglomerated, dense and homogeneous in chemical composition (Boulc'h et al., 2001). Particles size distribution was studied by laser granulometry. The profile shows a narrow distribution of sizes with mean value of around $800 \mathrm{~nm}$. Stoichiometry of powder was found to be similar to the precursor solution as measured by chemical analyses. Materials were characterized by a high purity with a quantity of silicium less than $0.1 \mathrm{wt} \%$ and only traces of hafnium. Raman spectrometry reveals tetragonal zirconia.

In the diffraction pattern, the peak broadening is due to three factors: strains, faulting and crystalline domain size. If we assume that the $2.5 \mathrm{Y}_{2} \mathrm{O}_{3}-\mathrm{ZrO}_{2}$ crystals are free of strains and faulting, peak broadening is only due to crystalline domain size. The domain size is calculated by the Scherrer formula (Warren, 1969):

$$
\mathrm{L}=(0.9 \lambda) /\left(\theta * \cos \theta_{0}\right),
$$

where $\mathrm{L}$ is the crystalline domain size (in $\mathrm{nm}$ ), $\lambda$ the wavelength (in $\mathrm{nm}$ ), $\theta$ the full width at half maximum (FWHM - in radian) and $\theta_{0}$ the diffraction angle $\left(^{\circ}\right.$ ). $\mathrm{L}$ is considered as an average crystal dimension perpendicular to the reflecting planes.

Yet, an additional instrumental broadening, arising from slit width, penetration in sample and imperfect focusing, is generally observed. Therefore, a standard sample, in which the particle size is large enough to eliminate all particle-size broadening, is used in order to correct the instrumental broadening. The standard peak is recorded under the analyzed sample conditions. Thus, the Scherrer formula is considered as:

$$
\begin{gathered}
\mathrm{L}=(0.9 \lambda) /\left(\Delta \theta * \cos \theta_{0}\right) \text { with } \\
\Delta \theta=\left(\mathrm{FWHM}_{\text {sample }}^{2}-\mathrm{FWHM}_{\text {standard }}^{2}\right)^{1 / 2} .
\end{gathered}
$$

This simple approximation is valid as long as the crystalline domain size is smaller than $100 \mathrm{~nm}$.

Powder X-Ray diffraction was carried out using a Siemens D500 $\theta / 2 \theta$ diffractometer with the BraggBrentano geometry from 20 to $80^{\circ}\left(0.04^{\circ}\right.$ in 20 step, 10 s counting time) equipped with a rear monochromator $\left(\mathrm{CuK}_{\alpha}\right.$ radiation, $\left.\lambda=1.5406 \AA\right)$. FWHM was determined by deconvolution of pseudo-Voigt shaped peaks with Profile Fitting software (Diffract-AT, Socabim, Paris).
Under HRTEM conditions, depending on the crystallographic orientation with respect to the electron beam, crystalline domains may be imaged as a dots lattice or more frequently as a fringe domain. Such fringe domains are sufficient to reveal the existence of crystalline domains and to measure their sizes. However, on the $2.5 \mathrm{Y}_{2} \mathrm{O}_{3}-\mathrm{ZrO}_{2}$ particles, thickness variation and domain overlapping lead to quite noisy images making difficult the measurements of fringe domains size on the HRTEM images. In such case, the geometrical phase analysis method, developed by Hytch, provides a method of interest to measure the crystalline domain size (see for instance Hytch and Gandais, 1995). The method can be summarized as follows. Basically, a fringe domain corresponds to a spatial frequency $(\mathrm{g})$ and a constant phase. Therefore, if a Fourier filtering followed by phase extraction is applied to a HRTEM image, a crystalline domain is identified by a constant phase area for a selected $\mathrm{g}$ vector.

The main advantage of the phase image method is that the crystalline domain appears as an homogeneous area characterized by an almost constant gray level. The rest of the particle which does not correspond to the selected $\mathrm{g}$ vector is defined by a very rapidly oscillating contrast on the map phase. Such both different behaviors simplify the image analysis processing further carried out to determine the size distribution.

Transmission electron microscopy observations were carried out on a Jeol 3010 microscope operating at $300 \mathrm{kV}$ (point resolution $\mathrm{r}=0.25 \mathrm{~nm}$ ). Powder was deposited on carbonated copper grid.

\section{RESULTS}

\section{XRD}

XRD pattern was characterized by broad peaks: indeed, 200, 202, 113 and 004 are not well resolved, as shown in Fig. 1. The absence of splitting in two diffraction lines, characteristic of tetragonal structure, may be attributed to small crystalline domain size.

Such XRD pattern is consistent with both tetragonal and cubic zirconia. Therefore, Raman spectrometry was a necessary preliminary step in order to conclude that $2.5 \mathrm{Y}_{2} \mathrm{O}_{3}-\mathrm{ZrO}_{2}$ powder, prepared by spraypyrolysis, crystallized into the tetragonal phase.

The Scherrer formula was applied to 111 peak. Indeed, this latter presents the maximum of intensity and is well-resolved in contrast to the broad 200,202,113 and 004 peaks. As detailed in Fig. 1, an 
average crystalline domain size of $6 \mathrm{~nm}$ was obtained from the broadening of the tetragonal zirconia peak compared to the 111 line of high-purity silicon, used as standard reference.

The $6.4 \mathrm{~nm}$ value belongs to the Scherrer formula validity domains. In order to corroborate the small domain size given by XRD interpretation and test the hypotheses relative to defects and strains, HRTEM observations were investigated.

\section{HRTEM AND IMAGE ANALYSIS}

Fig. 2a gives a typical HRTEM image of a $2.5 \mathrm{Y}_{2} \mathrm{O}_{3}-\mathrm{ZrO}_{2}$ nanoparticle. Crystalline domains appear as fringe domains. Each nanoparticle consists in several single crystalline domains. The $0.29 \mathrm{~nm}$ fringe spacing corresponds to the 111 planes of the tetragonal zirconia structure.

Fast Fourier Transform (FFT) of the image and phase extraction were carried out using the Semper software. Due to the small domain size and the image noise, the FFT gives a quite poor quality image. On the FFT, some spots located on a ring can be detected. Each spot corresponds to a $g$ vector, characteristic of crystalline domains whose the (111) planes are parallel to the electron beam.

Figs. $2 b$ and $2 c$ show the phase images which are obtained for two different $\mathrm{g}$ vectors selected among the diffraction ring. It is worth noting the ability of phase images to reveal small domains $(7 \mathrm{~nm})$ located within a $50 \mathrm{~nm}$ particle.

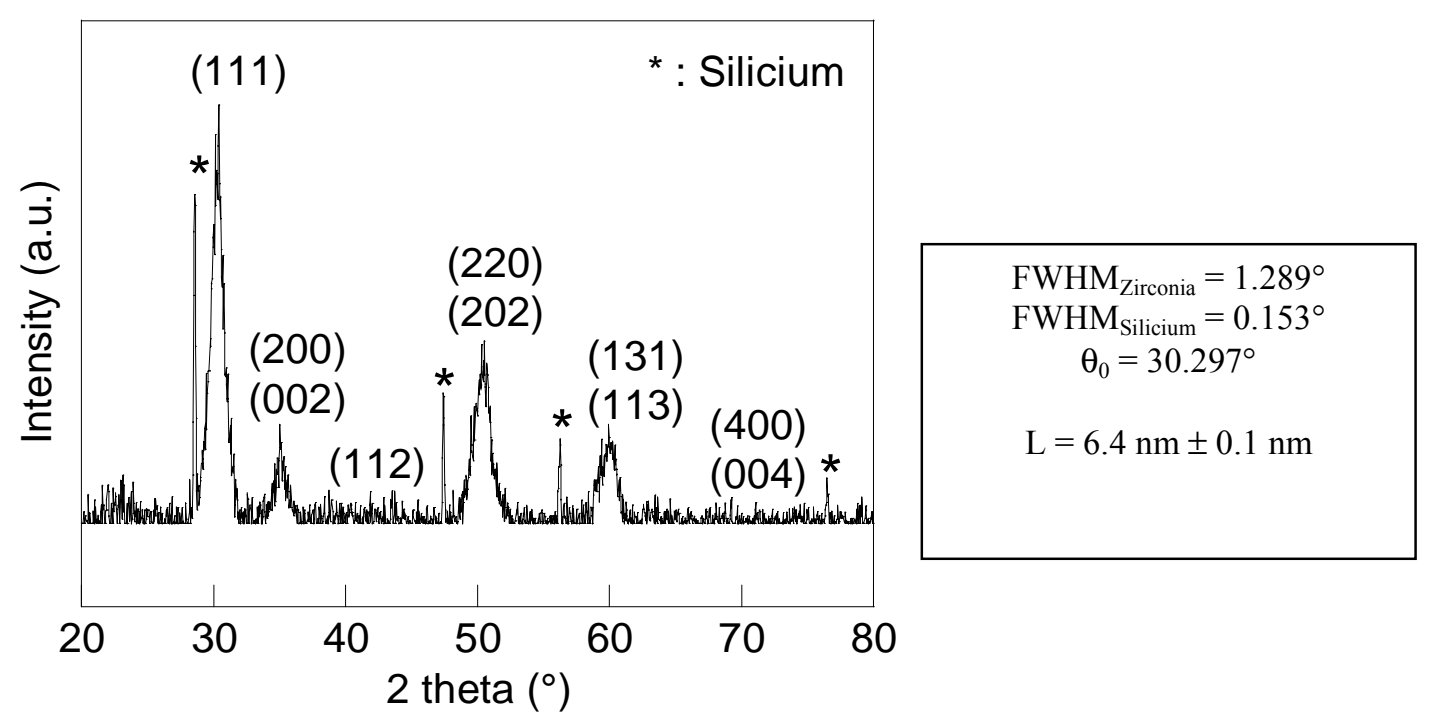

Fig. 1. X-Ray diffractogram of $2.5 \mathrm{Y}_{2} \mathrm{O}_{3}-\mathrm{ZrO} \mathrm{O}_{2}$.

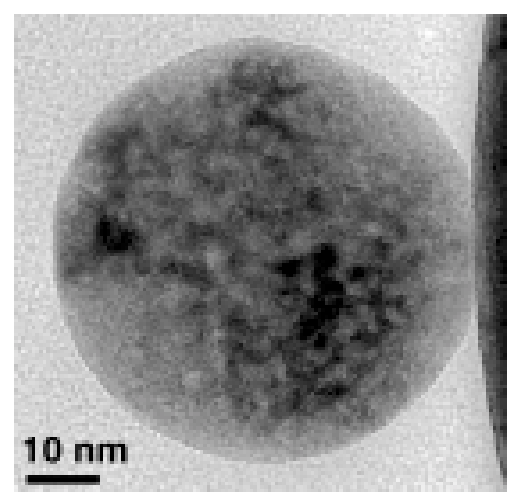

a)

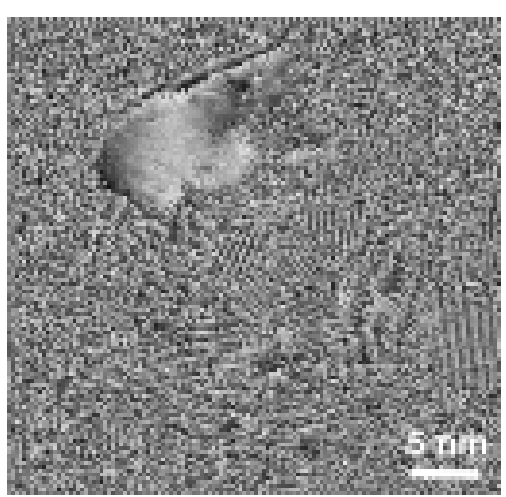

b)

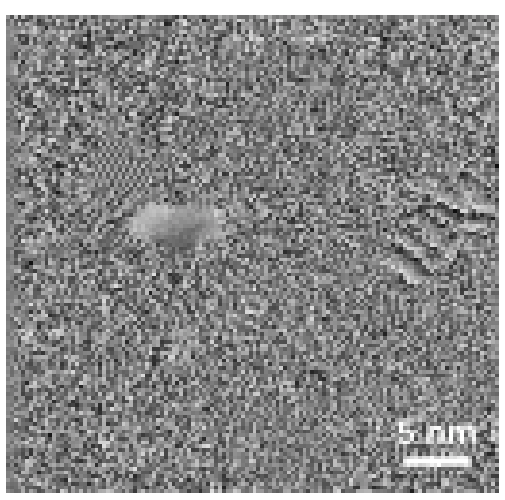

c)

Fig. 2. a) HRTEM image of $\left.2.5 \mathrm{Y}_{2} \mathrm{O}_{3}-\mathrm{ZrO}_{2}, b\right)$ and c) Phase images. 
Besides the homogeneous gray domains, the phase images may show some stripe domains. These latter correspond to crystalline domain characterized by a g' vector slightly different from the selected vector (typically g'-g $=1 / \mathrm{D}$ where $\mathrm{D}$ is the stripe width). Such stripe domains could be used for studying domain size and orientation relationship between domains. However, in the present work, we have only used the homogeneously gray domains to further determine the domain size distribution.

As illustrated by Fig. 2a, the magnification selected for imaging a $50 \mathrm{~nm}$ size particle are not appropriate to put in evidence clearly the fringes. Therefore, the phase image are particularly interesting since, at similar magnification, the particle as well as the fringe domains are imaged.

Moreover, phase images allow to have an information on the location of small and large domains. For instance, the large domains were systematically observed at the periphery of the particle. Such information might be of interest regarding the mechanisms of formation of crystalline domains in the $2.5 \mathrm{Y}_{2} \mathrm{O}_{3}-\mathrm{ZrO}_{2}$ particles (Messing et al., 1993).

The phase images are further analyzed with the Aphelion image analysis software (ADCIS). Figure 3a shows the image analysis result. Crystalline domain is characterized by a white domain whereas the rest of the particle is confused in a black one.

About 70 phase images were examined. Individual crystalline domain sizes $\mathrm{L}$ were defined as being equal to $\left(\mathrm{L}_{1} * \mathrm{~L}_{2}\right)^{1 / 2}, \mathrm{~L}_{1}$ and $\mathrm{L}_{2}$ correspond respectively to, the height and the width of the homogeneous gray domain. The error on $\mathrm{L}_{1}$ and $\mathrm{L}_{2}$ measurement is estimated to $0.3 \mathrm{~nm}$. This estimation is obtained by comparison of fringe domains in HRTEM images and the domain on the binary images. The small error is due to the limited number of operations in image analysis (Threshold, Erode and Dilate sequences). Size distribution function was deduced for the number $n_{i}$ of particles of size $L_{i}$.

Domain size distribution is shown in Fig. 3b. The crystalline domain size ranges from $5 \mathrm{~nm}$ to $22 \mathrm{~nm}$ with an average value of about $12 \mathrm{~nm}$.

\section{DISCUSSION}

An average crystalline domain size of $6 \mathrm{~nm}$ was calculated by the Scherrer formula. A $12 \mathrm{~nm}$ value was measured by HRTEM observations. Each technique does not measure the same length since the morphology, in particular, is not taken into account in the X-Ray method. The point here is rather to discuss on the possibilities and limits of XRD and HRTEM techniques in order to use their complementary nature to carry out efficiently further investigations.

HRTEM and image analysis provide direct information on the projection of domains on the observation plane and therefore allow to measurements without hypothesis on the domain shape. The main limit arises from the selection of particles observed by transmission electron microscopy. On one hand, usually, the thickness which is transparent to accelerated electrons and appropriate for HRTEM imaging is in the 10-100 $\mathrm{nm}$ range depending on materials and voltage. In the present case, it was observed that the fringe domains can be imaged only in particles with diameter below $50 \mathrm{~nm}$. Since the mean size of particles is $800 \mathrm{~nm}$ according to the granulometry results, it means that only the smallest particles were investigated by HRTEM. On the other hand, the too small crystalline domains embedded in a particle may be impossible to image. Such effect

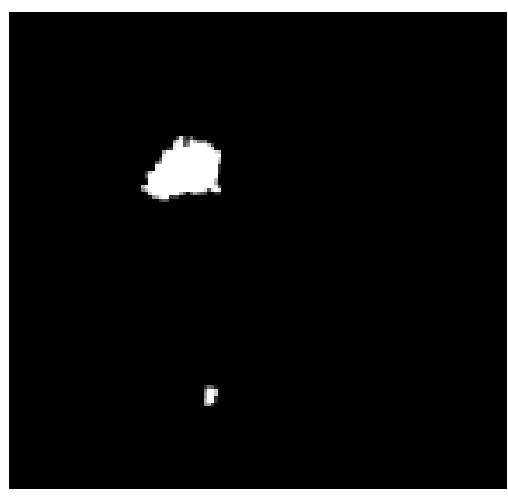

a)

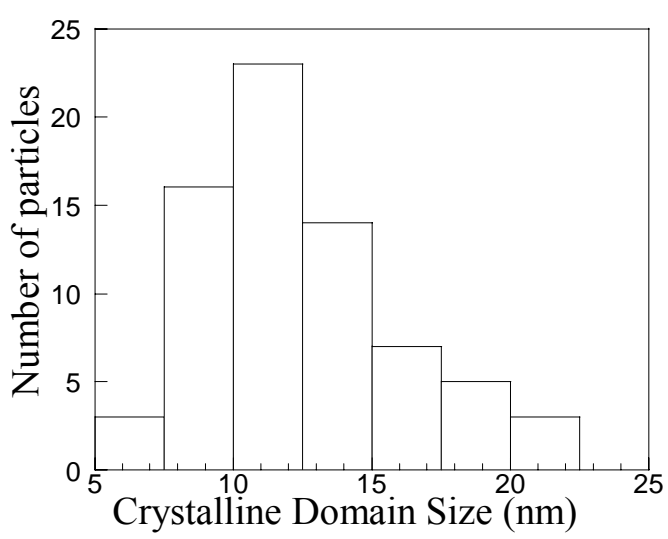

b)

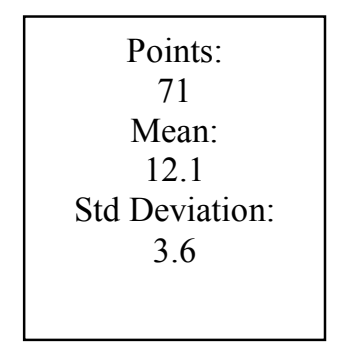

Fig. 3. a) Image analysis, b) Domain size distribution. 
will be all the more pronounced that the particles are large and the domains small, experimentally domains smaller than $5 \mathrm{~nm}$ were not observed. Moreover, depending on their position in a particle, the small domains may be more or less easy to image. These limitations are all due to the fact that TEM provides a projected image of a bulk samples, unfortunately the projection effect cannot be corrected by appropriate image analysis procedure.

Therefore, our measurements derived from HRTEM are representative of the smallest particles (i.e. $<50 \mathrm{~nm}$ ) and of crystalline domains greater than $5 \mathrm{~nm}$, contrary to, X-Ray diffraction which provides information on the whole system.

However, the Scherrer formula, as applied above, did not take in account possible defects and strains. In fact, according to the phase images, there are no dislocations or faults within the particles but strains are present. Indeed, Hytch et al. (1998) have shown that, on a crystalline domain phase image, defects produce discontinuities while strains give rise to slow continuous variations of the gray level. In the present case, the phase images were characterized by gray domain without discontinuities but a more or less important variation of the phase across the domain was observed. The phase image in Fig. $2 b$ is typical of the existence of strain within the crystalline domains.

\section{CONCLUSION}

Because of the beneficial effects of size reduction on application properties of yttria doped zirconia, fine scale characterization has to be carried out. Usually, the size of crystalline domains of the Y-TZP particles is calculated from X-Ray diffraction using the Scherrer formula, which gives quite simply and rapidly an average crystalline domain size. However, strains and defects are not taking in account in this simple approximation.

As illustrated in the present work, HRTEM images provide a valuable complementary analysis since the morphology of domains and their location within particles are evidenced. Moreover, when HRTEM images are associated to the phase image analysis, the domain size distribution is obtained quite directly. The essential drawback of the HRTEM method is that this measure is only representative of the small particles.
The complementary nature of both methods was here used to test the validity of the Scherrer formula hypothesis and to envisage future experiments. Firstly, it is worth noting that XRD and HRTEM analysis are consistent. Indeed, mean size of the same order of magnitude is obtained by both methods, i.e. the domain size of the $2.5 \mathrm{Y}_{2} \mathrm{O}_{3}-\mathrm{ZrO}_{2}$ powder belongs to the $10 \mathrm{~nm}$ scale. Secondly, the HRTEM image analysis indicates strains and absence of defects. Further X-Ray experiments are now in progress in order to evaluate the strain contribution. Once the question of strains solved, the next step will be to determine the distribution of domain size from X-Ray diffraction experiments.

\section{ACKNOWLEDGEMENT}

It is a pleasure to thank Dr. Françoise Bley (LTPCM) for her help in XRD discussion.

\section{REFERENCES}

Boulc'h F, Dessemond L, Fouletier J, Djurado E (2001). Physico-chemical characterization of highly pure nanocrystalline doped TZP. J Eur Ceram Soc, In press.

Djurado E, Meunier E (1998). Synthesis of doped and undoped nanopowders of tetragonal polycrystalline zirconia (TZP) by spray-pyrolysis. J Solid State Chem 141:191-8.

Djurado E, Bouvier P, Lucazeau G (2000). Crystallite size effect on the tetragonal-monoclinic transition of undoped nanocrystalline zirconia studied by XRD and Raman spectrometry. J Solid State Chem 149:399-407.

Hytch MJ, Gandais M (1995). Quantitative criteria for the detection and characterization of nanocrystals from high resolution electron microscopy images. Philos Mag A 72:619-34.

Hytch M, Snoeck E, Kilaas R (1998). Quantitative measurement of displacement and strain fields from HREM Micrographs. Ultramicroscopy 74:131-46.

Messing GL, Zhang SC, Jayanthi GV (1993). Ceramic powder synthesis by spray-pyrolysis. J Am Ceram Soc 76:2707-25.

Tsukuma K, Kubota Y, Tsukidate T (1984). Thermal and mechanical properties of $\mathrm{Y}_{2} \mathrm{O}_{3}$-Stabilized tetragonal zirconia polycrystals. In: Claussen N, Ruhle M, Heuer A, eds. Advances in ceramics science and technology of zirconia II. Ohio: The American Ceramic Society, Inc. Columbus, 12:382-3.

Warren BE (1969). Diffraction by imperfect crystals. In XRay Diffraction. New York: Dover Publications, Inc., 251. 